\title{
How does the oncogene astrocyte elevated gene-1 (AEG-1) augment glioma progression?
}
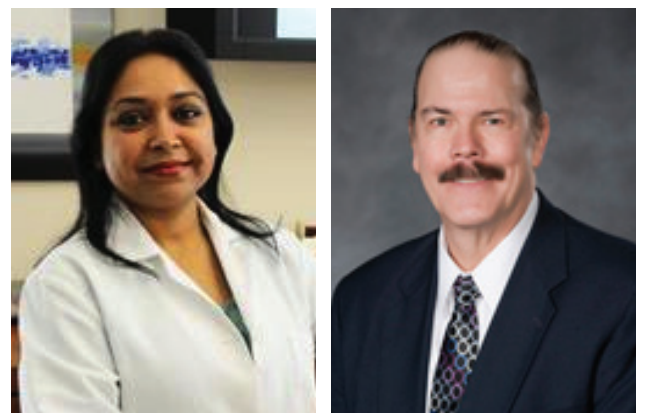

"The multifaceted role of AEG-1 on glioma progression implies that AEG-1 inhibition would be an appropriate end point to counter the pathogenesis of this dismal disease, particularly in conjunction with other current treatment modalities."

Luni Emdad ${ }^{\ddagger 1,2,3}$, Bin Hu', Timothy P Kegelman', Swadesh K Das ${ }^{1,2,3}$,

Devanand Sarkar ${ }^{1,2,3}$ \& Paul B Fisher ${ }^{*, \neq, 1,2,3}$

Gliomas are common primary brain tumors in adults that are for the most part lethal. Recent studies suggest that gliomas can arise from adult neural stem cells or multipotential neural progenitor cells that exist in the subventricular and subgranular zone [1]. According to the WHO, gliomas are classified into four malignancy grades (I-IV) based on their histological features; among these, grade I and II are considered low-grade gliomas with better prognosis, while glioblastoma multiforme ([GBM], malignant glioma, grade IV) has the worst prognosis. Patients diagnosed with malignant glioma display a median survival of approximately 12-15 months even with multimodal therapeutic approaches including maximal tolerated surgery, chemotherapy and radiotherapy [2]. Gliomas exhibit a strong propensity to invade healthy brain tissue by migrating through the convoluted extracellular spaces of the brain parenchyma, and eventually develop tumors at distant locations. Due to this invasive nature of the human malignant glioma, it is difficult to define borders between normal brain tissue and the tumor, which leads to incomplete surgical excision of the tumors. As such, radiotherapy or chemotherapy following surgery as combination therapies are standard of care for malignant glioma management [2]. Malignant gliomas are highly heterogeneous both genetically and pathologically, which makes the selection of diagnostic markers or targeted therapies challenging. The Cancer Genome Atlas (TCGA) network has established three essential signaling pathways that are important for malignant glioma progression including RTK/RAS/PI3K, p53 and Rb pathways [3]. Additionally, several hallmark tumorigenesis features are relevant for glioma progression, including uncontrolled proliferation, invasion, angiogenesis and chemo-radio resistance. Consequently, it is imperative to define and understand the

\section{KEYWORDS}

- AEG-1• angiogenesis • invasion - malignant glioma $\bullet$ progression

'Department of Human and Molecular Genetics, School of Medicine, Virginia Commonwealth University, Sanger Hall, Room 11-005, 1101 East Marshall Street, Richmond, VA 23298, USA 


\section{"AEG-1 not only promotes tumor progression but also contributes to chemoresistance."}

essential genetic elements controlling glioma progression. This information can be further exploited to identify new diagnostic/prognostic/therapeutic targets that will not only enhance survival of glioma patients, but will also provide new strategies to develop potent antiglioma drugs.

Astrocyte elevated gene-1 (AEG-1)

$A E G-1$ was first identified in 2002 as an elevated expressed gene in HIV-1/gp-120/TNF- $\alpha$ treated astrocytes [4]. In 2004, Brown and Ruoslahti identified a lung homing peptide in $A E G-1$, termed metadherin (MTDH), through a phage expression library of cDNA from a mouse model of lung metastasis of breast carcinoma that was overexpressed in metastatic breast cancer and promoted the homing of breast cancer cells to the lungs [5]. In the same year, the mouse-rat ortholog of $A E G-1$ was also discovered to encode the lysine-rich CEACAM-1 co-isolated protein (LYRIC) in tight junction of polarized epithelial cells [6], and as a novel transmembrane protein that resides in the endoplasmic reticulum, cytoplasm, nucleus and nuclear envelope [7]. AEG-1 overexpression is prevalent in a diverse array of human cancers and correlates with poor prognosis $[8,9]$. Gain-of-function (GOF) and lossof-function (LOF) research in vitro and in vivo showed that $A E G-1$ is involved in multiple pathological processes of human cancer, including proliferation, invasion, angiogenesis and metastasis via regulation of several important cellular signaling pathways.

\section{AEG-1 \& malignant glioma}

\section{- Expression analysis}

We first reported AEG-1 as a potential progression marker of malignant glioma [10], followed by reports from others and us establishing $A E G-1$ as a prominent oncogene in malignant glioma [10-12]. We demonstrated that AEG-1 is overexpressed in more than $90 \%$ of human malignant glioma samples as compared with normal human brain. By employing GOF and LOF experiments, we documented that AEG-1 regulates glioma migration, invasion and angiogenesis. In another study [11], Liu et al. performed a comprehensive analysis of 296 human glioma tissues and demonstrated that the expression of AEG-1 was notably upregulated and correlated with the clinicopathological grades of gliomas. Finally, in a recent study [12] from our group immunohistochemical analysis revealed a high correlation between expression of AEG-1 and Akt 2 in clinical glioma samples further confirming our earlier observations. Mining TCGA database analysis, we also confirmed the clinical significance of these two key molecules relative to glioma patient survival, for example, higher expression was associated with poorer survival.

\section{- Prosurvival role of $A E G-1$ \& signaling mechanisms}

Kang et al. [13] demonstrated the first link between $A E G-1$ expression and cancer progression. They documented that overexpression of $A E G-1$ significantly promoted anchorage-independent growth, a hallmark of the cancer phenotype. Subsequently, $A E G-1$ was established as an oncogene where overexpression in cloned rat embryonic fibroblasts (CREF) cells induced tumor formation in nude mice [14]. In another study, Lee et al. [15] showed that $A E G-1$ overexpression in primary human fetal astrocytes (PHFA) inhibited serum starvation induced apoptosis by activating the Ras and PI3K/Akt signaling pathways. AEG-1 regulation of Akt signaling pathways was shown by several other groups to be critical in cell proliferation, invasion, apoptosis resistance, chemoresistance and angiogenesis in multiple cancer contexts [16]. AEG-1 was shown to crosstalk with several signaling pathways to regulate cancer cell proliferation and promote glioma survival. We recently investigated the potential crosstalk between AEG-1-Akt 2 and NF- $\mathrm{KB}$ signaling, in malignant glioma [12]. By using an IKK inhibitor CAPE, we confirmed that CAPE modestly affected AEG-1-Akt2 signaling suggesting that NF- $\mathrm{KB}$ activation by AEG-1 is independent of Akt activation. AEG-1 was also shown to directly interact through its internal domain with the $\mathrm{PH}$ domain of $\mathrm{Akt} 2$, a major driver in gliomagenesis. Conditional expression of the Akt2-PH domain combined with AEG-1 silencing significantly enhanced survival in an orthotopic mouse model of human glioma [12].

\section{- AEG-1 \& invasion}

Glioma cell invasion is an integral component of glioma progression/pathogenesis and involves a complex multistep process, including tumor cell communication/adhesion with the extracellular matrix (ECM) and with adjacent cells, tumor cell migration and ECM degradation by proteases secreted by glioma cells. AEG-1 
regulates glioma invasion by modulating several proinvasive molecules and signaling pathways involved in this process. In malignant glioma cells, knockdown of $A E G-1$ results in invasion inhibition associated with a decrease in MMP-2 and MMP-9 activities [10]. Further mechanistic studies found that $A E G-1$ contributed to the invasive phenotype of glioma cells through transcriptional upregulation of MMP-9, one of the major mediators of glioma invasiveness [11]. One of the key molecular mechanisms by which AEG-1 promotes invasion and migration is by activating the NF- $\kappa \mathrm{B}$ pathway. AEG-1 directly binds to NF- $\kappa \mathrm{B}$ p 65 and acts as a linker between histone acetyltransferase CBP and p65 in malignant glioma [17]. Inhibition of NF- $\mathrm{KB}$ abolished AEG-1-induced enhanced migration and invasion in glioma cells. In a recent study, we showed that AEG-1 forms a novel complex with Akt2 in malignant glioma and disruption of AEG-1Akt2 interaction reduced AEG-1-mediated invasion [12].

\section{- AEG-1 \& angiogenesis}

Another feature of glioma progression is neovascularization/angiogenesis. Gliomas express high levels of proangiogenic and angiogenic factors including Angiopoietins (Ang-1 and 2) and their receptors, Tie-2, and VEGF and its receptors, VEGFRs. AEG-1 was shown to be involved in regulation of these proangiogenic molecules in glioma cells. Overexpression of $A E G-1$ in CREF cells resulted in highly angiogenic tumors in nude mice with enhanced expression of proangiogenic molecules, for example, MMP-2, CD31 (neovascularization marker), Ang1 and HIF1- $\alpha$. Additionally, when AEG-1 was overexpressed in glioma cells, it upregulated the expression of HIF1- $\alpha$ and also modulated VEGF promoter activity in a PI3K/Akt-dependent manner [14]. Interestingly, knocking down Tie-2 with siRNA significantly abrogated AEG-1-induced VEGF reporter activation in $\mathrm{H} 4$ glioma cells further supporting the proangiogenic role of AEG-1 in human glioma.

\section{- AEG-1 \& nutrient/oxygen deprivation}

In a recent study, AEG-1 expression was elevated during cellular stress, hypoxia and glucose deprivation in gliomas in an ROS-dependent manner [18]. Additionally, AEG-1 activated AMPK and its downstream targets in glioma cells, thus maintaining adequate ATP and promoting glioma proliferation. AMPK is also important for autophagy regulation and in this context AEG-1 was found to induce protective autophagy in immortalized PHFA cells in an AMPK-dependent manner [19].

\section{- AEG-1 \& chemoresistance}

AEG-1 not only promotes tumor progression but also contributes to chemoresistance. Previously, we demonstrated that AEG-1 KD in glioma cells facilitates chemosensitivity [10]. In a recent study from our group, we showed that AEG-1 and Akt-2 form a novel interaction complex, which is critical for regulation of cell survival, proliferation and invasion [12]. AEG-1 overexpression significantly improved overall cell viability in U87 and U251 cells upon TMZ treatment. In contrast, Akt2-PH, significantly decreased cell viability by itself, and also reduced the prosurvival effects of AEG-1 when treated with TMZ. These results indicate that $\mathrm{AEG}-1 / \mathrm{Akt} 2$ interaction is important for glioma cell survival under stress.

\section{- AEG-1 \& glutamate toxicity in glioma}

Glioma cells are capable of releasing large amounts of glutamate in vitro and in vivo. Overexpression of $A E G-1$ in glioma reduces expression of the glutamate transporter, EAAT2, resulting in glutamate-induced excitotoxicity [20]. Glutamate plays a prominent role in glioma pathogenesis affecting cell proliferation and migration. When astrocytes are treated with high concentrations of glutamate AEG-1 expression is enhanced [Emdad et al. Unpublished data]. Thus, a positive feedback loop exists between glutamate and AEG-1 potentially resulting in glioma progression by regulating invasion and migration.

Finally, AEG-1 plays a pivotal role in augmenting glioma progression by regulating several critical activities including survival, invasion, angiogenesis, chemoresistance and other processes. Preclinical studies utilizing RNAi approaches confirm improved overall survival in animal models of glioma. The multifaceted role of AEG-1 on glioma progression implies that AEG-1 inhibition would be an appropriate end point to counter the pathogenesis of this dismal disease, particularly in conjunction with other current treatment modalities. An alternate approach would be to develop small molecule drugs capable of disrupting the interaction of AEG-1 with other key driver molecules thereby functioning as novel therapeutics for malignant glioma.
“AEG-1 plays a pivotal role in augmenting glioma progression by regulating several critical activities including survival, invasion, angiogenesis, chemoresistance and other processes." 
Financial \& competing interests disclosure Research support was provided in part by NIH-NCI Grant R01 CA134721 and developmental funds from the VCU Massey Cancer Center (PBF). DS is a Harrison Scholar in the VCU Massey Cancer Center. PBF holds the Thelma Newmeyer Corman Chair in Cancer Research in the VCU Massey Cancer Center. The authors have no other relevant affiliations or financial involvement with any organization or entity with a financial interest in or financial conflict with the subject matter or materials discussed in the manuscript apart from those disclosed.

No writing assistance was utilized in the production of this manuscript.

\section{References}

1 Cuddapah VA, Robel S, Watkins S, Sontheimer H. A neurocentric perspective on glioma invasion. Nature Rev. Neurosci. 15(7), 455-465 (2014).

2 Wen PY, Kesari S. Malignant gliomas in adults. N. Engl. J. Med. 359(5), 492-507 (2008).

3 Wang $\mathrm{H}, \mathrm{Xu} \mathrm{T}$, Jiang $\mathrm{Y}$ et al. The challenges and the promise of molecular targeted therapy in malignant gliomas. Neoplasia 17(3), 239-255 (2015).

4 Su ZZ, Kang DC, Chen Y et al. Identification and cloning of human astrocyte genes displaying elevated expression after infection with HIV-1 or exposure to HIV-1 envelope glycoprotein by rapid subtraction hybridization, rash. Oncogene 21(22), 3592-3602 (2002).

5 Brown DM, Ruoslahti E. Metadherin, a cell surface protein in breast tumors that mediates lung metastasis. Cancer Cell 5 (4), 365-374 (2004).

6 Britt DE, Yang DF, Yang DQ et al. Identification of a novel protein, LYRIC, localized to tight junctions of polarized epithelial cells. Experimental Cell Res. 300 (1), 134-148 (2004).

7 Sutherland HG, Lam YW, Briers S, Lamond AI, Bickmore WA. 3D3/lyric: a novel transmembrane protein of the endoplasmic reticulum and nuclear envelope, which is also present in the nucleolus. Experimental Cell Res. 294(1), 94-105 (2004).

8 Sarkar D, Fisher PB. AEG-1/MTDH/LYRIC: Clinical significance. Adv. Cancer Res. 120, 39-74 (2013).

9 Huang Y, Li LP. Progress of cancer research on astrocyte elevated gene-1/Metadherin (review). Oncol. Letters 8(2), 493-501 (2014).

10 Emdad L, Sarkar D, Lee SG et al. Astrocyte elevated gene-1: a novel target for human glioma therapy. Mol. Cancer Therap. 9(1), 79-88 (2010).

11 Liu L, Wu J, Ying Z et al. Astrocyte elevated gene-1 upregulates matrix metalloproteinase-9 and induces human glioma invasion. Cancer Res. 70(9), 3750-3759 (2010).

$12 \mathrm{Hu}$ B, Emdad L, Bacolod MD et al. Astrocyte elevated gene-1 interacts with Akt isoform 2 to control glioma growth, survival, and pathogenesis. Cancer Res. 74(24), 7321-7332 (2014).

13 Kang DC, Su ZZ, Sarkar D, Emdad L, Volsky DJ, Fisher PB. Cloning and characterization of HIV-1-inducible astrocyte elevated gene-1, AEG-1. Gene 353(1), 8-15 (2005).

14 Emdad L, Lee SG, Su ZZ et al. Astrocyte elevated gene-1 (AEG-1) functions as an oncogene and regulates angiogenesis. Proc. Natl Acad. Sci. USA 106(50), 21300-21305 (2009).
15 Lee SG, Su ZZ, Emdad L, Sarkar D, Franke TF, Fisher PB. Astrocyte elevated gene-1 activates cell survival pathways through PI3K-Akt signaling. Oncogene 27(8), 1114-1121 (2008).

16 Emdad L, Das SK, Dasgupta S, Hu B, Sarkar D, Fisher PB. AEG-1/MTDH/LYRIC: signaling pathways, downstream genes, interacting proteins, and regulation of tumor angiogenesis. Adv. Cancer Res. 120, 75-111 (2013)

17 Sarkar D, Emdad L, Lee SG, Yoo BK, Su ZZ, Fisher PB. Astrocyte elevated gene-1: far more than just a gene regulated in astrocytes. Cancer Res. 69(22), 8529-8535 (2009).

18 Noch E, Bookland M, Khalili K. Astrocyteelevated gene-1 (AEG-1) induction by hypoxia and glucose deprivation in glioblastoma. Cancer Biol. Ther. 11(1), 32-39 (2011).

19 Bhutia SK, Kegelman TP, Das SK et al. Astrocyte elevated gene-1 induces protective autophagy. Proc. Natl Acad. Sci. USA 107(51), 22243-22248 (2010).

20 Lee SG, Kim K, Kegelman TP et al. Oncogene AEG-1 promotes glioma-induced neurodegeneration by increasing glutamate excitotoxicity. Cancer Res. 71(20), 6514-6523 (2011). 